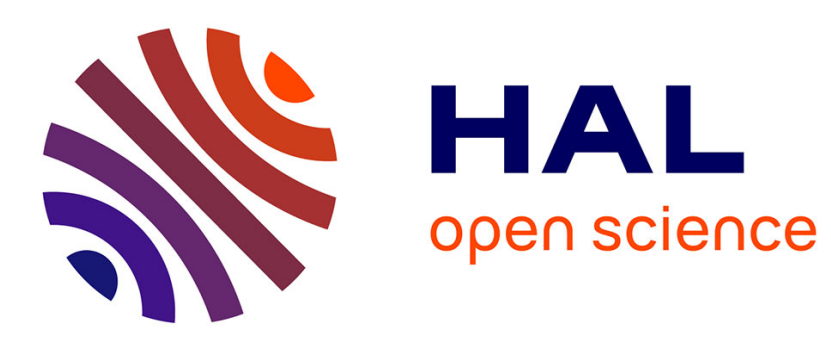

\title{
Localiser les enregistrements et les stimulations électriques du cerveau par la stéréotaxie
}

\author{
Jean-Gaël Barbara
}

\section{To cite this version:}

Jean-Gaël Barbara. Localiser les enregistrements et les stimulations électriques du cerveau par la stéréotaxie. Bulletin de la Shesvie, 2018. halshs-03090594

\section{HAL Id: halshs-03090594 \\ https://shs.hal.science/halshs-03090594}

Submitted on 29 Dec 2020

HAL is a multi-disciplinary open access archive for the deposit and dissemination of scientific research documents, whether they are published or not. The documents may come from teaching and research institutions in France or abroad, or from public or private research centers.
L'archive ouverte pluridisciplinaire HAL, est destinée au dépôt et à la diffusion de documents scientifiques de niveau recherche, publiés ou non, émanant des établissements d'enseignement et de recherche français ou étrangers, des laboratoires publics ou privés. 
Pour citer ce texte : J.G. Barbara, 2018, « Localiser les enregistrements et les stimulations électriques du cerveau par la stéréotaxie », Bulletin de la Shesvie, 25, 2018, 169-186, ISBN : 978-2-84174-907-2.

- version auteur -

\section{Localiser les enregistrements et les stimulations électriques du cerveau par la stéréotaxie}

Jean-Gaël Barbara est neuroscientifique et historien des sciences au CNRS. Il est spécialisé dans l'histoire des neurosciences et a publié La Naissance du Neurone, Vrin, 2010 et Le Paradigme neuronal, Hermann, 2010.

\section{Résumé}

On aborde ici la question des relations épistémologiques entre les pratiques des lésions expérimentales, de stimulation et d'enregistrement des activités électriques du cerveau, et celles concernant le recueil de données anatomiques et physiologiques, ou encore celles acquises chez l'animal et l'Homme, par le biais incontournable de la question des localisations des sites d'action dans ces procédures expérimentales par la technique de la stéréotaxie. On montre que l'histoire du développement de la stéréotaxie permet de mettre au jour de telles relations et de comprendre aussi bien la construction épistémologique de l'interdisciplinarité prônée par les neurosciences que les relations qu'il existe entre ces différentes approches par les techniques cliniques modernes telles que l'électroencéphalographie, la magnétoencéphalographie, la stimulation cérébrale profonde, la stimulation magnétique transcrânienne ou la neuroimagerie.

\section{Summary}

We investigate here the epistemological relations between the lesional studies of the nervous system, electrical stimulations and recordings of the brain, as well as those between anatomical and physiological data, or animal and clinical studies. Our approach is based on the study of the essential question dealing with the localization of such procedures by stereotaxy. We here show that the history of stereotaxy clearly shows such relations and enables us to understand the epistemological construct of the interdisciplinarity of the new neuroscience programme, as well as those relations established between modern clinical techniques of electroencephalography, magnetoencephalography, deep brain stimulation, transcranial magnetic stimulation or neuroimaging.

Les techniques actuelles d'enregistrement des activités électriques et de stimulation électrique du cerveau, comme l'électroencéphalographie, la magnétoencéphalographie et la stimulation 
cérébrale profonde ou la stimulation transcrânienne, sont à la fois l'objet de développements techniques et scientifiques importants dans le cadre des neurosciences, mais aussi d'enjeux fondamentaux dans la compréhension des mécanismes cérébraux et dans le traitement des pathologies neurologiques.

Or il n'est pas toujours aisé d'interpréter et de comprendre les relations épistémologiques qui existent entre les mises en œuvre de ces différentes techniques, ou encore leurs relations avec différents modèles du fonctionnement cérébral, comme le modèle du cerveau électrique et son implémentation par la théorie chimique de la neurotransmission au cours des années 1950. On peut par exemple demander si ce sont les mesures électriques ou les stimulations localisées qui déterminent les connaissances premières à partir desquelles d'autres techniques viennent en aide pour approfondir les modèles et quelle est la place des connaissances anatomiques dans ces enquêtes, aussi bien que les relations entre les données cliniques et celles recueillies chez l'animal.

Une histoire des techniques d'enregistrement et de stimulation électriques du cerveau peut aider à débrouiller quelques-uns de ces rapports, et on abordera ici en particulier la question sousjacente fondamentale de l'importance de localiser avec précision les sites d'enregistrement et de stimulation électriques, mais d'un point de vue historique, c'est-à-dire en fonction de l'évolution des différentes techniques associées à la physiologie cérébrale.

Si l'histoire des techniques dans celle des neurosciences revêt une importance capitale, les historiens de la biologie ont le plus souvent sous-estimé son étude, comme se plaisait à l'écrire Gerald L. Geison en $1995^{1}$. Cela est particulièrement visible dans notre cas des techniques de repérage de l'espace cérébral, dont la première, la stéréotaxie, repose sur un instrument de physiologie complexe, l'appareil de stéréotaxie, dont il est rarement fait mention précisément dans les études d'histoire des sciences et qui est également souvent non mentionné explicitement dans les articles scientifiques eux-mêmes qui l'utilisent.

Tout cela demande des précisions et une étude de quelques détails choisis. Un appareil de stéréotaxie est un cadre métallique mobile, positionnable sur la tête d'un sujet ou d'un animal, qui constitue à la fois un système de contention du crâne et un espace de référence utilisant un système de coordonnées polaires pour maintenir en position une électrode afin d'ensuite la guider en la faisant glisser vers l'intérieur de l'espace cérébral en un lieu précis. Un micromanipulateur et un guide de maintien de la microélectrode s'adaptent en effet sur ce cadre et sont positionnables dans l'espace de référence en choisissant certaines coordonnées, de manière à ce qu'ils permettent la descente dans le cerveau de la microélectrode en un point précis, déterminé par ses coordonnées. Cette technique est utilisée en neurochirurgie pour atteindre des zones du cerveau de manière précise et permet aussi chez l'animal de définir la position d'une microélectrode dans une certaine structure cérébrale pour procéder à un enregistrement ou une

\footnotetext{
${ }^{1}$ « Nous souhaitons insister sur [...] le rôle [...] des instruments [et sur] l'absence de reconnaissance de [leur] importance [...] à la hauteur de leur sophistication », D.J. Kevles, G.L. Geison, "The Experimental Life Sciences in the Twentieth Century", Osiris, 1995, 10, Constructing Knowledge in the History of Science, p.120.
} 
procédure de lésion, de stimulation, de refroidissement ou encore d'injection d'un composé chimique.

Les histoires de la stéréotaxie qu'on rencontre le plus souvent se confondent avec celle de la neurochirurgie, alors que la stéréotaxie a été utilisée au départ dans l'expérimentation animale comme technique fondamentale d'exploration. Chez l'animal comme chez l'Homme, on distingue l'exploration fonctionnelle - par exemple par des stimulations électriques par électrodes implantées en vue d'une intervention chirurgicale chez un patient épileptique -, et l'exploration des activités et l'étude de leurs relations entre régions du cerveau pour déterminer les organisations fonctionnelles des systèmes nerveux étudiés.

L'étude de l'émergence historique de la technique de stéréotaxie, et de ses liens complexes avec les techniques de physiologies cérébrales animale et humaine, permettra de comprendre l'importance du repérage dans l'espace cérébral en physiologie, et l'analyse épistémologique précisera les liens entre les approches de mesure et de stimulation électrique du cerveau dans la création de modèles cérébraux.

\section{L'instrument stéréotaxique de Victor Horsley et Robert H. Clarke (1905)}

Ce nouvel instrument de physiologie, l'appareil stéréotaxique, est né au tournant du $\mathrm{XX}^{\mathrm{e}}$ siècle, de la volonté de pouvoir localiser précisément des lésions réalisées expérimentalement chez l'animal à l'intérieur du cervelet, selon une approche lésionnelle classique, en vue de déterminer les fonctions des différentes régions du système nerveux, dans un contexte dans lequel les connaissances anatomiques avaient pris une certaine avance sur l'investigation physiologique du cerveau, demeurée plus grossière, en raison de la taille importante des électrodes et des possibilités alors très limitées d'amplification des signaux électriques par des électromètres ${ }^{2}$.

Ce problème fondamental de localisation se posa en réalité dès les premières études lésionnelles du système nerveux réalisées dans l'Antiquité, notamment avec les expériences de vivisection de l'École d'Alexandrie reprises par le médecin Claude Galien (II' siècle ap. J.-C.). Il s'agissait de déterminer la fonction d'un organe par l'examen des troubles physiologiques déterminés par sa blessure accidentelle ou son ablation expérimentale par vivisection. Galien devint maître dans cette technique expérimentale et il put ainsi analyser par cette méthode les fonctions des ventricules du cerveau et celles de la moelle épinière ${ }^{3}$.

\footnotetext{
2 Anonymous, Section of Physiology, Br. Med. J., 1906, 2, part 1, p.633. R.H. Clarke, V. Horsley, On a method of investigating the deep ganglia and tracts of the central nervous system (cerebellum), Br. Med. J. 1906, 2, p.1799-1800.

${ }^{3}$ On cite un extrait d'un texte de Galien : «Une lésion du ventricule postérieur n'empêche pas la vision. Chez l'animal, cela se vérifie par le fait qu'il ferme les yeux lorsqu'on approche un objet devant lui. En revanche, l'effet sur la vision est évident lorsqu'on comprime les ventricules antérieurs. Tu verras alors comment l'animal cligne des yeux, surtout si tu approches un objet de ses yeux, même après qu'on ait exposé à la vue le quatrième ventricule. Si tu t'approches de l'animal dans ces conditions et comprimes une partie de ses deux ventricules antérieurs, quelle que soit la partie, là où j'ai situé la racine des deux nerfs optiques, alors l'animal cesse de cligner avec ses deux yeux même quand on approche quelque objet de ses pupilles, et tout l'aspect de l'œil du côté où est le ventricule comprimé ressemble aux yeux d'un homme aveugle.», Galien, Administrations anatomiques, IX, 11-12. trad. fr. in Mirko D. Grmek, Le chaudron de Médée. L'expérimentation sur le vivant dans l'Antiquité, Institut Synthelabo, Coll. Les empêcheurs de
} 
Ce type d'étude, repris dans le contexte de la physiologie expérimentale, à partir des années 1830, utilisa alors des compressions et des destructions de plus en plus fines, surtout à partir de 1837, lorsque fut découvert le « nœud vital » par Pierre Flourens et César Julien Jean Legallois, un centre nerveux du bulbe si petit qu'une aiguille pouvait le détruire d'un seul coup. Dès lors, il fut admis que la découverte des propriétés et des fonctions des centres nerveux reposait sur la précision des lésions et leur localisation fine, ce que recommandait Pierre Flourens ${ }^{4}$.

C'est donc la quête éternelle d'une telle précision lors des vivisections qui poussa Robert Henry Clarke (1850-1926), soixante-dix ans plus tard, à construire, suite à une pneumonie durant laquelle il fut immobilisé et libre de son temps, à la demande de Victor Horsley (1857-1916), son ami et chef de file de l'école de neurologie britannique, un instrument de repérage des positions du cerveau ${ }^{5}$. Clarke souhaitait régler ce problème de précision en particulier dans ses études lésionnelles sur le cervelet réalisées en collaboration avec Horsley et alors que certains physiologistes avaient développé précédemment des instruments moins précis ayant le même objectif, comme celui par exemple construit en 1873 par Charles Dittmar (1843-1917) dans le laboratoire de Carl Ludwig à Leipzig ${ }^{6}$.

Dès le début de ces nouvelles recherches utilisant un appareil de localisation, on assiste à plusieurs croisements épistémologiques entre des perspectives d'étude différentes. Un premier est réalisé entre l'analyse physiologique et les données anatomiques. Il devient en effet possible de déterminer à l'avance la position anatomique précise de l'électrode et de la vérifier post-mortem par l'histologie. Un second se situe entre l'exploration physiologique du cerveau des animaux et les possibilités d'exploration cérébrale chez l'Homme. Un troisième enfin se développe d'une part, entre les expériences de lésion et de stimulation et, d'autre part, entre les expériences de lésion et d'enregistrement d'activité. Dans tous les cas, c'est la stéréotaxie qui permet ces croisements, car on peut corréler les données, par exemple en positionnant à la même place une électrode d'enregistrement et une aiguille destinée à produire une lésion.

Le croisement des données issues du cerveau des animaux et les possibilités d'exploration cérébrale chez l'Homme est réalisé massivement durant les années 1950, mais il est manifeste dès les commencements de la stéréotaxie en 1906. Clarke souhaite en effet déjà rapidement appliquer sa technique à l'Homme - il dépose d'ailleurs un brevet -, mais il se fâche avec son ami Horsley à ce sujet, pour lequel l'instrument doit d'abord seulement être destiné à l'expérimentation animale.

penser en rond, Paris, 1997, p.157-159, cité in Armelle Debru, «L'expérimentation sur le cerveau et le système nerveux dans l'Antiquité », La Lettre des Neurosciences, 2007, 32, p.3-8.

${ }^{4}$ Pierre Flourens écrivait en 1824 : «On verra pourquoi Rédi, et Zinn, et Haller, et Lorry, et les autres, ont observé des phénomènes si confus sur les animaux qu'ils mutilaient si aveuglément, et sans savoir sur quelles parties portaient leurs mutilations, et surtout sans avoir, par une analyse expérimentale préalable, déterminé l'expression propre de chacune de ces parties », Pierre Flourens, Recherches expérimentales sur les propriétés et les fonctions du système nerveux dans les animaux vertébrés, Paris, Crevot, 1824, p. 58.

${ }^{5}$ Louise H. Marshall, Horace W. Magoun, "The Horsley-Clarke Stereotaxic Instrument: The Beginning”, Carrier, 1990, 27, p.15-19.

${ }^{6}$ P. Blomstedt, M. Olivecrona, A. Sailer, M.I. Hariz, "Dittmar and the history of stereotaxy; or rats, rabbits, and references", Neurosurgery, 2007, 60, p.198-201. 
Les études d'expérimentation animale se multiplient alors, avec le prêt par Clarke de son instrument à Ernest Sachs (1879-1958) pour des études sur le thalamus (1907-1909) ${ }^{7}$, et avec les travaux de S.A. Kinnier Wilson (1878-1937) (1914), de F.J.F. Barlington, de Paul Karplus et d'Alois Kreidl, pour des expériences sur l'hypothalamus (1909-1911) ${ }^{8}$; puis il semble que l'instrument se soit perdu sûrement en raison de sa difficulté d'utilisation et de l'absence de transmission des procédés de mise en œuvre.

\section{L'école de neuroanatomie de Chicago de Stephen Ranson}

À partir de 1924, Stephen Ranson, le renommé professeur d'anatomie de la Northwestern University de Chicago, reprend ce type d'étude et fait construire son propre instrument selon les plans de l'appareil de Clarke de $1908^{9}$. C'est le point de départ de l'importante école de neuroanatomie fonctionnelle de Chicago dont les études furent basées sur des lésions très bien circonscrites, puis des stimulations. Par exemple, on rechercha le rôle de l'hypothalamus dans la régulation des fonctions de l'hypophyse, tel que le contrôle de la diurèse et de la quantité d'eau de l'organisme. Cette étude démarra en réalité en reprenant une recherche de Charles Sherrington sur le rôle de l'hypothalamus dans le contrôle de la posture, avec intégration des données anatomiques et physiologiques. Ensuite, l'élève de Ranson, Horace Magoun réalisa des études avec Ranson, puis seul, sur les fonctions du cervelet, l'hypothalamus, les centres respiratoires de la moelle qui s'étendent jusqu'à la formation réticulée ou encore le cortex olfactif. Dans ces études, Magoun fit d'ailleurs souvent référence à des travaux préliminaires et inachevés de Robert Clarke.

En 1934, Ranson et Magoun obtiennent un soutien de la fondation Rockefeller pour qui la stéréotaxie représentait un progrès fondamental qu'il convenait de saluer et de soutenir à sa juste valeur. Car en effet la stéréotaxie expérimentale chez l'animal entraîne une sorte de révolution, dans la précision des nouvelles recherches sur les localisations des fonctions du cerveau, soutenue par les institutions. C'est aussi, et surtout au début, une révolution technique par les croisements épistémologiques entre différentes approches expérimentales (lésions, stimulations, enregistrements), et les correspondances qu'on peut établir entre anatomie et physiologie, et entre l'animal et l'Homme.

En avril 1935, un officier de la fondation Rockefeller visite l'institut de Ranson et estime que le travail le plus intéressant qui y est réalisé est celui qui tire le meilleur parti de l'instrument de Horsley et de Clarke ${ }^{10}$. La précision de ces études est en effet extraordinaire, inférieure à un millimètre, et la nouvelle aire du Brain Mapping débute, avec également rapidement des implications psychiatriques.

\footnotetext{
${ }^{7}$ E. Sachs, "On the structure and functional relations of the optic thalamus", Brain, 1909, 32, p.95-186.

${ }^{8}$ Cité in S. Finger, "Origins of neuroscience: a history of explorations into brain function", Oxford, Oxford University Press, 2001, p.285.

${ }^{9}$ Voir Tara H. Abraham, "Transcending disciplines: Scientific styles in studies of the brain in mid-twentieth century America”, Studies in History and Philosophy of Biological and Biomedical Sciences, 2012, 43, p.552-568, p.557.

${ }^{10}$ Ibid.
} 
Cette nouvelle ère de l'étude du cerveau se développe à l'heure de l'émergence de la nouvelle neurophysiologie qui est une électrophysiologie neuronale, c'est-à-dire l'étude des activités électriques des fibres nerveuses isolées et des neurones, suite aux progrès de l'amplification électronique des signaux électriques, par les lampes triodes, puis les oscillographes cathodiques ${ }^{11}$. De sorte que cette nouvelle physiologie cérébrale, dont les mécanismes sont localisables par la stéréotaxie, devient une neurophysiologie cérébrale fondamentale et clinique, avec aussi l'émergence du nouveau domaine de l'électroencéphalographie durant la même période des années 1930. Ainsi se crée par la stéréotaxie et la neurophysiologie élémentaire et globale (électroencéphalographie), un nouveau modèle de cerveau électrique, avec ses implications psychiatriques - à l'ère des traitements par chocs électriques - et philosophiques - avec le thème de la « pensée électrique ».

\section{Extension progressive des études de stéréotaxie}

$\mathrm{Au}$ cours des années 1930, bien d'autres groupes utilisent désormais la stéréotaxie qui s'est démocratisée progressivement, notamment dans le sillage de Sachs et de Barlington. C'est ainsi qu'on met au point de nouveaux appareils adaptés pour travailler sur le rat et sur le chat, avec la publication d'atlas pour ces espèces, mentionnant pour chaque région cérébrale les coordonnées polaires correspondantes.

Il devient alors possible de localiser avec une grande précision les mesures d'enregistrement électrique ou encore de température. C'est ainsi que l'axonologiste, Ralph Gerard, utilise la technique pour réaliser une cartographie des températures du cerveau d'un chat qui annonce les principes de l'imagerie cérébrale par la cartographie d'un indice relié au métabolisme cellulaire ${ }^{12}$.

Le Géorgien, Ivane Beritashvili, fut à la fois le premier à utiliser l'oscillographe cathodique en URSS et l'électroencéphalographie, mais aussi à introduire l'appareil de Horsley-Clarke en 1938$1939^{13}$.

Au fil du temps, l'association de ces techniques devient la règle aux États-Unis et définit ce que Louise Marshall décrit comme la neurophysiologie de style américain ${ }^{14}$, alors qu'en Europe la stéréotaxie est encore peu ou pas présente.

Aux États-Unis, on assiste dans ce contexte à un retour des cartographies fonctionnelles après celles de Fritsch, Hitzig et Ferrier - et à une reprise des thèmes de recherche de la fin du $\mathrm{XIX}^{\mathrm{e}}$ siècle et du début du $\mathrm{XX}^{\mathrm{e}}$ siècle, période durant laquelle on avait assisté à un passage de la stimulation électrique à la lésion électrolytique. Cependant l'étude des organisations

\footnotetext{
${ }^{11}$ Voir J.G. Barbara, La Naissance du neurone, Paris, Vrin, 2010.

12 Ralph Gerard, H.M. Serota, "Localized thermal changes in the cat's brain", J. Neurophysiol., 1937, 1, p.115-124.

${ }^{13}$ I. Beritashvili, encore dénommé I. Beritov ou J. Beritoff (1884-1974).

${ }^{14}$ L.H. Marshall, "Instruments, techniques, and social units in American neurophysiology, 1870-1950" in G.L. Geison (Ed.), Physiology in the American context: 1850, Bethesda, MD, American Physiological Society, 1987, p. 351-369.
} 
fonctionnelles concerne alors, d'une manière nouvelle, les structures profondes, comme cela avait été commencé lors des travaux de Horsley et de Clarke.

Pour Louise Marshal, la stéréotaxie permet aussi de recentrer les recherches sur une physiologie des centres nerveux, à partir d'une physiologie nerveuse antérieure plus centrée sur l'étude des sensations comme la vision, le tact ou le codage intensité-fréquence des sensations dans la neurophysiologie d'Edgar Adrian ${ }^{15}$.

\section{Les nouveaux développements des années 1940}

Au cours des années 1940, la stéréotaxie continue son développement et elle est adaptée à l'Homme par le neurophysiologiste allemand Ernst Spiegel et le neurochirurgien Henry Wycis, avec le projet de réaliser des lésions dans les noyaux de la base pour traiter certains troubles moteurs, puis, plus tard, dans le noyau thalamique dorsomédian dans le traitement des psychoses $^{16}$.

Les études s'affinent chez l'animal lorsqu'on envisage de manière plus systématique d'étudier la fonction des régions profondes du cerveau par l'étude de leurs relations fonctionnelles avec d'autres structures. C'était d'ailleurs ainsi que Ranson avait débuté ses travaux sur l'hypothalamus, car il n'était alors possible de découvrir ses fonctions qu'en étudiant ses liens fonctionnels avec par exemple l'hypophyse, en analysant l'effet d'une lésion de l'hypothalamus sur une fonction de l'hypophyse.

C'est ainsi que Magoun réalise des études fonctionnelles de certaines voies nerveuses, par exemple la voie cortico-bulbo-réticulaire. On peut également citer les travaux très précis de Bishop et O'Leary et ceux de Sachs à la Washington University. Mais c'est surtout la découverte du système réticulaire ascendant et sa fonction dans l'éveil par Moruzzi et Magoun en $1949{ }^{17}$ qui marque les esprits, grâce à l'utilisation centrale, non toujours remarquée, de la stéréotaxie, sur des animaux anesthésiés au chloralose.

Cette découverte est réalisée dans un contexte général aux États-Unis dans lequel de brillants jeunes chercheurs s'emparent de la nouvelle technique de la stéréotaxie qui paraît de plus en plus prometteuse et incontournable. Ainsi Jerry Lettvin se forme à la stéréotaxie à l'université de l'Illinois et rejoint le laboratoire de son professeur de psychiatrie, Warren $\mathrm{McCulloch}^{18}$. On peut citer parmi ces jeunes chercheurs, outre Lettwin et McCulloch, John N. Brookhart, Vernon Mountcastle, Wade H. Marshall ou encore Peter Bishop.

\footnotetext{
${ }^{15}$ Voir J.G. Barbara, 2010, op. cit.

${ }^{16}$ Jack D. Pressman, "Reflections on Neurosurgery in Philadelphia: Oral Histories With Frederick Murtagh and Henry Shenkin”, Interview with Dr. Fred Murtagh, Transactions \& Studies, Medicine \& History, série V, volume XII, n 1 , mars 1990, p.27-48.

${ }^{17}$ G. Moruzzi \& H.W. Magoun, "Brain stem reticular formation and activation of the EEG", Electroencephalogr. Clin. Neurophysiol., 1949, 1, p.455-473.

${ }^{18}$ En 1947, J. Lettvin et W. McCulloch réalisent un travail de stéréotaxie présenté sous forme de résumé à un congrès, mais Lettvin regrettera dans son autobiographie de ne pas l'avoir publié. J.Y. Lettvin, The path of suppression in the spinal grey matter, Fed. Proc., 1948, 7, p.71.
} 


\section{La stéréotaxie en France}

En 1948, le Français Henri Gastaut apprend la stéréotaxie à Montréal dans l'institut du neurochirurgien W. Penfield, avec Herbert Jasper. Puis il revient à Marseille et ramène une bourse Rockefeller, ainsi que du matériel de recherche, dont un appareil de stéréotaxie Horsley-Clarke. Mais au laboratoire de Gastaut, c'est Robert Naquet qui utilise le «Horsley-Clarke » et qui attire l'attention de la neurophysiologiste parisienne, Denise Albe-Fessard, avec laquelle il collabore à l'Institut Marey, par des allers-retours Marseille-Paris incessants, mais avec également Pierre Buser, pour des études sur le singe qui concernent l'implication des structures sous-corticales dans la réaction d'éveil, un thème proche des recherches de H. Magoun ${ }^{19}$. Naquet part ensuite lui aussi se perfectionner chez Herbert Jasper à Montréal.

En France également, le professeur de pathologie générale à la faculté de médecine de Paris, Alphonse Baudouin, et son neveu et élève Antoine Rémond, entrent en scène en 1947. Rémond réalise des travaux utilisant la stéréotaxie de 1950 à 1961. Puis Antoine Rémond utilise un appareil de stéréotaxie pour soigner des patients parkinsoniens, peut-être en reprenant les travaux de H. Narabayashi ; Rémond réalise des lésions en ciblant le pallidum avec 30 ans d'avance, selon son élève Bernard Renaud ${ }^{20}$.

\section{Des lésions aux enregistrements électriques (1948)}

Dans la physiologie de Haller, au XVIII ${ }^{\mathrm{e}}$ siècle, l'aiguille était un moyen de stimulation des tissus, puis elle devient un instrument de destruction d'un petit centre nerveux. Il y a en réalité une continuité épistémologique entre la stimulation et la destruction d'un circuit nerveux, car tandis que la destruction peut être obtenue par une stimulation forte qui réalise une perte de fonction, une stimulation modérée permet un gain de fonction, ou un renforcement de l'activité d'un centre nerveux.

Le déplacement épistémologique est beaucoup plus grand quand on utilise de fines aiguilles ayant une fonction d'électrode d'enregistrement des activités électriques. Et c'est en 1948 qu'apparaissent les premiers enregistrements électriques réalisés avec de fines électrodes dont la position d'implantation est contrôlée par stéréotaxie in vivo, puis vérifiée ensuite post mortem par l'histologie. Ces travaux minutieux sont engagés par trois équipes, celle de V. Mountcastle avec l'étude des représentations tactiles dans le thalamus, celle de H. Jasper dans

\footnotetext{
${ }^{19}$ Robert Naquet raconte : « [...] je me promenais avec mes chats dans leur panière, mon « Horsley-Clarke » et j'arrivais à Paris chez les Fessard en compagnie de Vigouroux [...] pour faire une démonstration à la Pitié-Salpêtrière au siège de la société d'EEG. Il y avait là mon ami Jean Scherrer, un excellent neurophysiologiste qui était allé lui aussi aux États-Unis et qui était en train d'installer son laboratoire de physiologie. On endormait nos chats appareillés avec leurs électrodes devant tout le monde, ce qui serait inconcevable aujourd'hui. Dans la salle il y aurait sûrement des défenseurs des animaux! C'était la grande époque de la neurophysiologie », entretien avec Robert Naquet (1921-2005) de J.-F. Picard du 21 janvier 1997.

${ }^{20}$ Voir C. Chérici, Barbara J.G., « EEG, trois lettres pour percer les mystères du cerveau », La revue pour l'Histoire du CNRS, 2007, 19, p.21-25.
} 
l'étude des connexions sous-corticales, celle de J. Brookhart avec l'étude d'unités pyramidales du bulbe, puis des unités dans le cervelet avec G. Moruzzi.

Il ne s'agit plus dans ces études de faire une lésion localisée, mais de produire l'indice d'une activité neuronale localisée, une activité électrique à l'échelle du neurone. Avec ces enregistrements d'activités neuronales, il est possible de déterminer les liaisons fonctionnelles directes, monosynaptiques, avec des mesures de délais synaptiques courts, qui ne peuvent être mis en évidence de façon sûre qu'en mesurant des activités de neurones uniques, ou de petits ensembles de neurones synchronisés. L'enregistrement unitaire localisé seul permet donc d'étudier plus sûrement les organisations fonctionnelles et la stéréotaxie permet de les étudier dans les structures profondes.

Au cours des études d'enregistrement d'activités neuronales dans la période précédente des années 1940, on utilisait des pénétrations peu profondes, comme par exemple celles de 1 à $2 \mathrm{~mm}$ pratiquées à la surface de l'encéphale de poissons par P. Buser avec un micromanipulateur ; P. Buser étudiait alors de façon descriptive, avec beaucoup d'incertitudes dans l'interprétation, des phénomènes d'inversion de dipôles interprétés comme des variations globales de polarisation entre les arborescences axoniques et dendritiques des neurones ${ }^{21}$.

\section{Vers les neurosciences (années 1950)}

Les études de stéréotaxie vont se développer de manière plus importante encore au cours des années 1950, dans des cadres interdisciplinaires mieux définis, des associations plus étroites avec la psychiatrie et la prise en compte du comportement animal, dans un cadre théorique modulé par l'adoption généralisée de la théorie chimique de la neurotransmission.

Par exemple, un rapport d'Alan Gregg de la fondation Rockefeller, datant de 1950, souligne en effet que la psychiatrie doit réunir l'anatomie et la neurophysiologie à la clinique, la neurochimie et la neurochirurgie ${ }^{22}$.

Or c'est précisément ce que permet la stéréotaxie qui représente alors non seulement une technique, mais surtout une modalité d'étude expérimentale qui s'immisce dans différents champs de recherche des neurosciences à venir, en tissant entre eux des possibilités de combinaison. La stéréotaxie permet en effet de combiner des techniques par l'association avec des procédés d'enregistrements, des procédés de libération contrôlée de substances pharmacologiques par iontophorèse à un endroit précis, ou des procédés de microstimulation ou de microrefroidissement. C'est à l'intérieur d'un tel cadre qu'Antoine Rémond associe également ces techniques dans des essais cliniques à la Salpêtrière pour tenter des traitements de la maladie de Parkinson.

\footnotetext{
${ }^{21} \mathrm{P}$. Buser, Analyse des réponses électriques du lobe optique à la stimulation de la voie visuelle chez quelques vertébrés inférieurs, thèse présentée à la Faculté des sciences de l'Université de Paris, pour obtenir le grade de docteur ès sciences naturelles, Paris, Masson, 1955.

${ }^{22}$ A. Gregg, Report, April $1^{\text {rst }}$ 1950, Rockefeller Foundation Trustees Bulletin, 1950, RAC.
} 
Les recherches sur l'animal permettent de poursuivre les études fines des organisations fonctionnelles des circuits neuronaux. Par exemple, H. Magoun étudie en 1951 le diffuse thalamic projection system. Mais tout change avec la découverte du «système activateur ascendant réticulaire » qui ouvre un nouveau champ de recherche sur l'éveil et la conscience. G. Moruzzi de Pise, qui a collaboré avec Magoun dans ces découvertes, poursuit l'étude de ces structures dans son laboratoire avec des enregistrements unitaires.

Si les études unitaires chez l'animal ne sont pas encore associées avec l'étude des comportements, on en prend le chemin avec la mise au point de techniques d'électrodes implantées. C'est dans le contexte de ces développements techniques que James Olds découvre en 1956 le «centre du plaisir », avec l'observation qu'un rat peut déclencher, par un levier, la stimulation de ce centre et ce d'une façon qui se renforce dans le temps. L'animal apprend à déclencher cette stimulation de manière répétée, car la stimulation conditionne son comportement et représente pour le rat une récompense et une source de plaisir.

On essaie en effet de travailler sur des animaux non anesthésiés ${ }^{23}$, et cela ouvre la voie à des corrélations plus nombreuses avec l'étude du comportement grâce à l'utilisation de conditionnements ${ }^{24}$, comme par exemple dans les travaux de E. Grastyán ${ }^{25}$. Un regain d'intérêt des savants occidentaux se manifeste pour ceux des pays de l'Est avec leurs traditions d'étude des conditionnements. Par exemple, les jeunes chercheurs français, Jean Massion et Gilbert Lelord, partent se former, au cours des années 1960, chez le neurophysiologiste polonais Jerzy Konorski. Ces collaborations aboutissent à la fin des années 1950, et au cours des années 1960, à rechercher des corrélats électrophysiologiques des conditionnements ${ }^{26}$.

En parallèle, les atlas de stéréotaxie se multiplient et concernent de nouveaux animaux comme les singes. On associe la stéréotaxie et la pharmacologie en développant des systèmes de microperfusion avec des canules, ce qui permet d'étudier la pharmacologie de certains phénomènes comme ceux associés à l'activation de la formation réticulée.

Ces études concernent alors de nouveaux modèles animaux, par exemple des modèles de maladies comme l'épilepsie, avec les techniques modernes d'enregistrement unitaire, même si les anciennes voies de recherche persistent, celles utilisant des lésions et des ablations, aidées d'une manière nouvelle par la stéréotaxie chez l'animal, avec une standardisation progressive des procédures et des instruments. On adopte ainsi des repères précis selon des critères internationaux et on prend l'habitude de mentionner dans les articles les coordonnées des «plans stéréotaxiques ».

\footnotetext{
${ }^{23}$ P.B. Bradley, J. Elkes, "A technique for recording the electrical activity of the brain in the conscious animal", Electroencephalography and Clinical Neurophysiology, 1953, 5, p.451-456.

${ }^{24}$ Voir par exemple, Henry Gluck,Vernon Rowland, "Defensive conditioning of electrographic arousal with delayed and differentiated auditory stimuli”, Electroencephalography and Clinical Neurophysiology, 1959, 11, p.485-496.

${ }^{25}$ E. Grastyán, K. Lissák, I. Madarász, H. Donhoffer, "Hippocampal electrical activity during the development of conditioned reflexes, Electroencephalography and Clinical Neurophysiology, 1959, 11, p.409-430.

${ }^{26}$ Par exemple, Eliot Hearst, Bernard Beer, Guy Sheatz, Robert Galambos, "Some electrophysiological correlates of conditioning in the monkey", Electroencephalography and Clinical Neurophysiology, 1960, 12, p.37-152.
} 


\section{Le grand développement de la stéréotaxie humaine (années 1950)}

C'est encore au cours des années 1950 que la stéréotaxie se développe massivement en neurologie, avec les techniques d'électrodes implantées - celles créées pour les animaux vigiles - qui sont utilisées chez 1'Homme ${ }^{27}$. En France Jean Talairach et Pierre Buser utilisent ces techniques à l'hôpital Sainte-Anne dans la recherche de foyers épileptiques par stimulation de la zone supposée du foyer à l'aide d'électrodes implantées, chez des patients présentant une épilepsie pharmacorésistante.

On peut aussi mentionner les tests de fonctionnalité concernant les zones corticales qu'on envisage d'extraire au cours de chirurgies du cerveau pour des ablations de tumeurs, pour éviter l'endommagement de certains centres importants comme l'aire du langage.

Dans tous les cas, on réalise ces tests avec consentement du patient et l'on envoie de légers courants dans une zone pour voir si cela produit une crise d'épilepsie ou si l'on stimule l'aire du langage. Dans cette première phase de test clinique de telles implantations, on étudie aussi leurs conséquences négatives possibles chez l'Homme.

Aussi ces utilisations courantes de la stéréotaxie chez l'Homme permettent de collecter des données et d'envisager la création d'atlas mentionnant les coordonnées polaires des aires cérébrales $^{28}$ avec la publication, en 1957, par l'équipe de Jean Talairach d'un atlas stéréotaxique de l'Homme ${ }^{29}$. En réalité, les recherches de stéréotaxie humaines sont engagées, on l'a vu, dès les années 1940, et la Fondation Rockefeller soutient d'ailleurs un autre projet d'atlas de stéréotaxie humaine dès 1952, qui voit le jour en $1959^{30}$. Selon Gordon Shepherd, l'atlas de J. Talairach fut plus largement adopté au point qu'il est encore utilisé comme référence pour l'analyse des données d'imagerie.

Au-delà de ces utilisations cliniques spécifiques et de la création d'atlas, la stéréotaxie se répand dans d'autres domaines de la clinique et intéresse de jeunes médecins et chercheurs qui désirent tous se former à la stéréotaxie dès le début des années 1950. Par exemple, après un séjour chez H. Magoun, Michel Jouvet improvise et construit en France lui-même un appareil peu précis en $1958^{31}$. Lors de son internat en neurochirurgie et en neurologie, il enregistre des réponses

${ }^{27}$ Par exemple, T. Okuma, Y. Shimazono, T. Fukuda, H. Narabayashi, "Cortical and subcortical recordings in nonanesthetized and anesthetized periods in man", Electroencephalography and Clinical Neurophysiology, 1954, 6, p.269-286.

${ }^{28}$ Voir C. Cherici, «La définition d'une entité clinique entre développements techniques et spécialisation médicale : épilepsie et épileptologie au XX ${ }^{\mathrm{e}}$ siècle », Revue d'Histoire des Sciences, 2010, 63, p.409-437 ; «L'année 1957 marque une amélioration du repérage anatomique stéréotaxique car la ligne CA-CP [commissure blanche antérieure et postérieure] est utilisée comme un système de référence commune à la ventriculographie, l'encéphalographie fractionnée et la téléventriculographie dont les résultats deviennent alors superposables. En 1958, paraît un ouvrage important de Jean Talairach et Pierre Tournoux intitulé L'Exploration chirurgicale stéréotaxique du lobe temporal dans l'épilepsie temporale. »

${ }^{29}$ J. Talairach, M. David, P. Tournoux, H. Corredor, T. Kvasina, Atlas d'anatomie stéréotaxique, Paris, Masson, 1957.

${ }^{30}$ Rockefeller report, 1952, p.85-86. Il s'agit du projet d'atlas humain du docteur Georges Schaltenbrand de 1'université de Wiirzburg (Allemagne), en collaboration avec le docteur Percival Bailey de l'université de l'Illinois. Cela donnera lieu à la publication de G. Schaltenbrand et P. Bailey, Introduction to Stereotaxis with an Atlas of the Human Brain, New York, Grune \& Stratton, 1959.

${ }^{31}$ Interview de Michel Jouvet, par S. Mouchet et J.-F. Picard, du 31 octobre 2007, à La Colombière, Sainte Croix, 01120, Montluel. 
évoquées visuelles sous-corticales à des flashs de lumière et il étudie le rôle de l'attention dans la discrimination $^{32}$. Cette période se caractérise par un croisement important des travaux cliniques et fondamentaux. Ainsi H. Jasper étudie des unités chez des patients parkinsoniens ${ }^{33}$, E.A. Spiegel, H.T. Wycis réalisent des enregistrements sur le thalamus chez l'Homme ${ }^{34}$, tandis qu'Antoine Rémond pratique des lésions du pallidum également chez des patients parkinsoniens.

\section{L'association aux études du comportement (années 1960)}

Les nouvelles électrodes implantées à demeure mises au point à la fin des années 1950 permettent de réaliser des enregistrements d'activité électrique chez l'animal libre de ses mouvements, avec des comportements observables, quantifiables et maîtrisés par des protocoles de conditionnement.

Par exemple, Pierre Buser utilise la stéréotaxie dans ses études utilisant des conditionnements opérants chez le chat avec Philippe Ascher, puis Arlette Rougeul. Avec Pierre Borenstein, Pierre Buser utilise la stéréotaxie pour étudier les réponses visuelles dans le thalamus.

Puis le grand tournant que prennent ces études est celui concernant les corrélats électrophysiologiques des conditionnements. Il s'agit de rechercher des corrélations entre certaines activités électriques et des comportements conditionnés. Si un comportement est conditionné, on peut alors déclencher l'enregistrement d'une zone du cerveau avec une électrode implantée au moment où le comportement est provoqué par conditionnement. C'est ainsi qu'Arlette Rougeul, l'épouse de Pierre Buser, étudie avec lui des rythmes attentionnels chez le chat; elle mesure ces rythmes au moment où le chat se fige devant une proie qu'on lui présente. Le chat « joue le jeu », car il a été entraîné par un conditionnement opérant. Cette technique s'applique aussi à des singes entraînés à réaliser des tests cognitifs, lorsqu'ils sont maintenus assis, avec des mesures par des électrodes implantées dans certaines zones du cerveau. C'est ainsi que le modèle électrique du cerveau qu'on décrivait initialement de manière globale par des mécanismes généraux de synchronisation d'activités élémentaires réalisant des intégrations successives des activités devient plus spécifique avec le développement de modèles de traitement des signaux sensoriels dans des circuits neuroniques hiérarchisés.

\section{La découverte des cellules de lieu de l'hippocampe : une recherche des années 1960}

L'aventure de la découverte des cellules de lieu de l'hippocampe démarra par l'observation d'une simple corrélation observée entre certaines activités unitaires et un élément environnemental simple, la localisation de l'animal dans un repère spatial. John O'Keefe et Jonathan

\footnotetext{
${ }^{32}$ M. Jouvet, J. Courjon, «Variation des réponses évoquées visuelles sous corticales au cours de l'attention chez l'homme », Rev. Neurol., 1958, 99, p.177-178.

33 H.H. Jasper, G. Bertrand, "Recording from microelectrodes in stereotaxic surgery for Parkinson's disease", J. Neurosurg., 1966, 24, p.219-221.

34 E.A. Spiegel, H.T. Wycis, "Thalamic recordings in man with special reference to seizure discharges", Electroencephalography and Clinical Neurophysiology, 1950, 2, p.23-27,
} 
Dostrovsky établirent ce type de corrélation dans un nombre limité de neurones hippocampiques ${ }^{35}$.

Ces neurones se caractérisaient en effet par leur particularité de décharger lorsque l'animal était dans un même lieu circonscrit - un champ de lieu ou place field - et celle d'être silencieux en dehors de ce lieu.

Pour réaliser de tels travaux, il fallait pouvoir enregistrer les activités unitaires de neurones de l'hippocampe avec un animal libre de ses mouvements, dans un espace limité et ouvert. D'autres recherches de ce type furent menées ayant pour thèmes par exemple des cartographies des champs récepteurs sensoriels ${ }^{36}$.

\section{Le Neuroscience Research Program}

Le premier programme de neurosciences, le Neuroscience Research Program (NRP), organisé par l'Américain Francis O. Schmitt est un mouvement scientifique fédérateur des années 1960 qui s'est donné pour mission de recenser les nouvelles tendances des études du système nerveux, en vue de développer de nouvelles interdisciplinarités.

Ce mouvement n'est pas resté étranger aux grands progrès de la neurochirurgie et de la stéréotaxie en particulier. Dans un bulletin du NRP, le NRP Bulletin publié en 1966, on trouve une grande synthèse sur le thème de la mémoire avec un rapport sur les expériences de neurochirurgie et les déficits de mémoire associés. Cette synthèse prône le rapprochement des connaissances de neurochirurgie, de psychiatrie, de neurologie et de psychologie expérimentale relatives à des déficits de mémoire observés chez des patients ayant subi des lésions chirurgicales réalisées comme moyen thérapeutique pour des indications neurologiques ou psychiatriques.

On voit bien comment le NRP essaya de brouiller les frontières entre les disciplines médicales en vue de comprendre les grandes fonctions du système nerveux, ce qui fut également réalisé en mettant en parallèle les recherches réalisées sur des animaux, comme les expériences citées dans le compte rendu du premier colloque du NRP sur le codage sensoriel chez des animaux curarisés étudiés par des électrodes implantées avec la stéréotaxie. Sont également citées des expériences sur le concept de drive, avec une tentative de synthèse des approches passées dans l'étude des lésions de l'hypothalamus et des comportements alimentaires. L'attention est toujours portée, comme chez Pierre Flourens déjà, vers la minutie des lésions et l'auteur n'hésite pas à faire un parallèle, dans le contexte de la biologie moléculaire naissante, entre des lésions microscopiques du système nerveux et les modifications moléculaires des immunoglobulines réalisées à cette période par G. Edelman.

\footnotetext{
${ }^{35}$ J. O'Keefe, J. Dostrovsky. The hippocampus as a spatial map. Preliminary evidence from unit activity in the freelymoving rat. Brain Res, 1971, 34, 171-175. Voir J.G. Barbara, Le Paradigme neuronal, pdf, p. 237.

${ }^{36}$ D.H. Hubel, "Single unit activity in lateral geniculate body and optic tract of unrestrained cats", J. Physiol., 1960, 150, p.91-104.
} 
Le NRP soutient également l'utilisation de la stéréotaxie dans tous les types d'approches comme l'étude pharmacologique de la mémoire en injectant des substances par stéréotaxie, avec ou sans lésion. Au cours du second colloque du NRP, on met en avant des travaux d'électroencéphalographie avec des électrodes implantées par stéréotaxie dans l'amygdale ou la formation réticulée, pendant une tâche cognitive, dans ce cas un jeu électronique.

On comprend donc que l'intérêt du NRP pour la stéréotaxie se développe dans un contexte dans lequel les techniques se diversifient et parviennent à s'associer librement en raison de leur complémentarité. Mais surtout, c'est l'association du comportement avec l'étude sur des animaux libres de leurs mouvements et le conditionnement qui permet des découvertes comme celle des cellules de lieu qui annonce dans sa méthode celle des neurones miroirs des années 1990. Ainsi les développements de la stéréotaxie caractérisent bien d'une certaine façon, et d'un certain point de vue, l'essor du mouvement des neurosciences et de manière plus globale tous les liens épistémologiques, avec tous les rapprochements progressifs de leurs données, des diverses approches expérimentales du système nerveux, lésions, stimulations, enregistrements, et leurs modèles associés et complémentaires du cerveau. Ces liens permettent d'envisager de la même manière les relations actuelles complexes qui existent entre l'enregistrement des activités électriques, les stimulations électriques et la création de modèles des fonctions du cerveau ${ }^{37}$.

\footnotetext{
${ }^{37}$ On pourra voir à ce sujet les analyses épistémologiques suivantes : J.G. Barbara, « Neurobiologie, histoire de la », « Réseaux de neurones, biologie », " Imagerie par résonance magnétique de diffusion (IRMD) », "Visualisation de l'activité du cerveau» in Encyclopaedia Universalis, en ligne. J.G. Barbara, "L'exploration des réseaux de neurones », in La science au présent, 2017, p.62-67. J.G. Barbara, "Voir le cerveau fonctionner », in Universalia 2018, Paris, Encyclopædia Universalis, 2018, p.296-303.
} 